\title{
Management problems in severe chronic inducible urticaria: Two case reports
}

\author{
ROXANA-SILVIA BUMBĂCEA ${ }^{1,2^{*}}$, CRISTINA-GEORGIANA DEACONU ${ }^{2}$ and ELENA CAMELIA BERGHEA ${ }^{3 *}$ \\ ${ }^{1}$ Department of Allergology and Clinical Immunology, 'Carol Davila' University of Medicine and \\ Pharmacy, 050474 Bucharest; ${ }^{2}$ Novo Medica, 050554 Bucharest; ${ }^{3}$ Allergology Outpatient Clinic, \\ The Clinical Emergency Hospital for Children 'M.S. Curie', 041434 Bucharest, Romania
}

Received August 19, 2018; Accepted February 25, 2019

DOI: $10.3892 /$ etm.2019.7651

\begin{abstract}
Chronic inducible urticaria (CIndU) is a subgroup of chronic urticaria which can cause severe quality of life impairment by their refractory forms. The recommended treatment approach in CindU is the same as that for chronic spontaneous urticaria (CSU). However, CIndU seem to be more resistant to standard doses of $\mathrm{H} 1$ antihistamines (AHs) and higher doses of AHs are required for symptom control. Omalizumab, a recombinant anti-IgE antibody, effectively treats CSU. Nevertheless, there is not enough evidence in patients with CIndU, especially in AHs resistant cases. This study analyzed 2 severe cases of CIndU (cold urticaria and symptomatic dermographism) with completely different response to omalizumab. We describe 2 patients with 2 subtypes of CIndU: one with severe cold urticaria (including anaphylaxis) and the other with severe extensive symptomatic dermographysm. In both cases, we performed complete positive and differential diagnostic work-up. Management strategies included first line and second line symptomatic therapy, but with no success in either case. Avoidance of eliciting triggers was difficult to achieve (occupational reasons). We decided to start omalizumab treatment, $300 \mathrm{mg}$ every 4 weeks for 6 months. The cold urticaria patient gained complete symptom relief 10 days after the first dose of omalizumab; the quality of life improved substantially with no side effects of the treatment. The urticaria factitia
\end{abstract}

Correspondence to: Dr Elena Camelia Berghea, Allergology Outpatient Clinic, The Clinical Emergency Hospital for Children 'M.S. Curie', 20 Bd Brancoveanu Constantin, 041434 Bucharest, Romania

E-mail: bcamelia@gmail.com

*Contributed equally

Abbreviations: CSU, chronic spontaneous urticaria; CIndU, chronic inducible urticaria, AHs, H1 antihistamines; OCS, oral corticosteroids; LTRA, leukotriene receptor antagonist

Key words: inducible urticaria, cold urticaria, symptomatic dermographysm, omalizumab patient showed no benefit of the add-on 5 months treatment with omalizumab. He refused the 6th dose of omalizumab due to the lack of response, and also cyclosporine, but he showed some benefits of oral corticosteroids. Although many clinical studies support the use of omalizumab in the treatment of patients with CIndU, we certainly need more data for prediction of a good clinical response.

\section{Introduction}

Chronic inducible urticaria (CIndU) is a subgroup of chronic urticaria, characterized by the presence of wheals (hives), angioedema, or both, over 6 weeks, triggered by a specific factor (1). They are divided in physical urticaria and nonphysical urticaria. The physical urticaria is induced by exogenous physical triggers: thermal triggers (cold, heat), solar radiation and mechanichal triggers (friction, pressure). In nonphysical urticaria (cholinergic, contact or aquagenic urticaria) the active and passive warming, contact with some substances or water are required. The recommended treatment approach in CIndU is the same as that for chronic spontaneous urticaria (CSU) (1-3). The first line treatment includes H1-antihistamines in standard doses or four-fold higher doses. Some patients do not respond even to high level of antihistamines. Omalizumab, a recombinant humanized monoclonal antibody against $\mathrm{IgE}$, can be an efficient add-on therapy in recalcitrant CSU (3). Some case reports and small case series offer limited data regarding the efficiency of omalizumab in CIndU.

The study was approved by the Ethics Commitee of Emergency Clinical Hospital for Children 'M.S. Curie' (Bucharest, Romania), and a signed informed consent was obtained from the patients or the guardians included in this study.

We present 2 severe cases of CIndU (one with severe cold urticaria and the other with severe extensive dermographism) with completely different response to omalizumab.

\section{Case reports}

Case 1. A 38-year-old woman presented with a seven months history of a severe cold urticaria. First symptoms occurred after swimming in the sea water during her summer holiday. She described wheals, chills and some dizziness which disappeared after warming. Her hives were transient, did not burn 
Table I. General characteristics of the cases with physical urticaria.

\begin{tabular}{|c|c|c|c|c|c|c|c|c|c|c|}
\hline Case & $\begin{array}{c}\text { Age } \\
\text { (years) }\end{array}$ & $\operatorname{Sex}$ & $\begin{array}{c}\text { Duration } \\
\text { of disease } \\
\text { (months) }\end{array}$ & $\begin{array}{l}\text { UAS7 at } \\
\text { baseline }\end{array}$ & $\begin{array}{l}\text { Type of } \\
\text { urticaria }\end{array}$ & $\begin{array}{c}\text { Total } \\
\text { IgE } \\
\text { (UI/ml) }\end{array}$ & $\begin{array}{l}\text { Previous } \\
\text { treatment }\end{array}$ & $\begin{array}{c}\text { Dose of } \\
\text { omalizumab }\end{array}$ & Duration & Effect \\
\hline 1 & 38 & $\mathrm{~F}$ & 7 & 42 & Cold & 828 & 4 AH1, 2 AH2, LTRA & $300 \mathrm{mg} / \mathrm{month}$ & 6 months & Yes \\
\hline 2 & 17 & M & 25 & 42 & Factitia & 348 & 4 AH1, AH2, LTRA, OCS & $300 \mathrm{mg} / \mathrm{month}$ & 5 months & No \\
\hline
\end{tabular}

F, female; M, male; AH1, H1-antihistamines; 4AH1, four-fold higher doses of AH1; AH2, H2-antihistamines; LTRA, leukotriene receptor antagonist; OCS, oral corticosteroids.

and did not leave bruising as they resolved. Shortly thereafter, in addition to the above symptoms, she began to have episodes of angioedema with shortness of breath and dysphonia and with severe dizziness after exposure to cold wind; all these episodes resulted in emergency room visits where epinephrine was administered with improvement in symptoms. During winter time, her symptoms were getting worse, with severely impaired quality of life and with need of interruption of her job of medical assistant. Her family history revealed a grandfather with asthma and her mother with Basedow's disease.

Through specific investigations a negative workup has been shown for mast cell disorders, hereditary angioedema, autoimmune diseases (including cryoglobulinemic vasculitis), infections (including hepatitis B and C), and food allergy, inhalant allergy. She had an intense positive ice cube test result and an increased level of total serum $\operatorname{IgE}(828 \mathrm{UI} / \mathrm{ml})$. Diagnosis of severe cold urticaria (including anaphylaxis) was established.

Initial treatment consisted of high-dose (up to 4-fold) of various $\mathrm{H} 1$ antihistamines (AHs) (desloratadine, bilastine, rupatadine) and $\mathrm{H} 2$ blockers (ranitidine) with further addition of leukotriene receptor antagonist (montelukast). Specific prophylactic measures have been respected (as much as possible). Despite this treatment, the response was poor, the patient being forced to stay at home, with warm clothing and still experienced some intermittent hives.

We decided to start on monthly omalizumab $(300 \mathrm{mg}$ ) injections. At this point, her urticaria activity score for the preceding 7 days (UAS7) was 42 (Table I). The benefit came very fast, from the first dose. Within 10 days after starting this medication, her wheals were controlled. The UAS7 fell to 28 (at first month evaluation) with a progressive decrease after each course (Fig. 1). No side effects were noted during her 6 courses of omalizumab. At the time of this case study, 10 months after the interruption of the omalizumab, she remains free of severe anaphylactic episodes, with only some mild hives triggered by specific cold circumstances, but she is still taking $\mathrm{H} 1$ and $\mathrm{H} 2$ antihistamines (maximum doses).

Case 2. The second case we report, is the case of a 17-year-old boy who came for a history of 25 months of severe symptomatic dermographism (urticaria factitia). The patient's complaints were severe itchiness and whealing in context of usual activities at home or at school (dressing, tight clothes, some physical activities), and in the last year also spontaneous urticaria added (urticarial plaques from time to time, without an obvious trigger, without drug-induced or food-induced

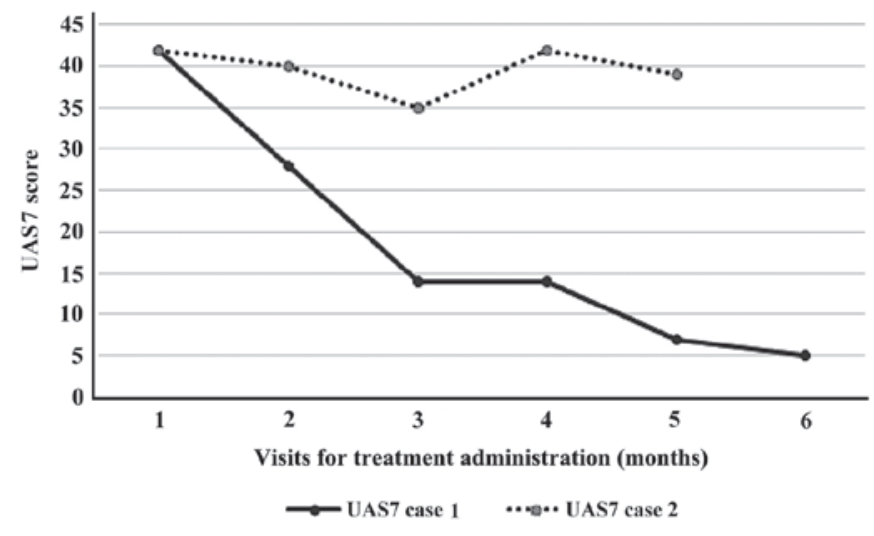

Figure 1. Development of the urticaria activity score over 7 days (UAS7) score for case 1 and 2 measured at each visit monthly.

exacerbation). In this condition, the patient described decreased school performance and negative impact on social life. The family history was negative for any significant disease. Differential blood count, ESR or CRP and the entire diagnostic workup (infections, autoimmune disorders, $\mathrm{IgE}$ mediated food and inhalant allergies) were in normal ranges, except for total IgE (348 UI/ml). The diagnosis of urticaria factitia was supported by the history and the skin reaction at the typical trigger (itching palpable wheals within $10 \mathrm{~min}$ after light stroking pressure with a wooden spatula on the volar forearm) (1). The treatment with various nonsedating antihistamines (bilastine, desloratadine, rupatadine, levocetirizine) in standard and up to four-fold higher doses, single or in combination with $\mathrm{H} 2$ blocker (ranitidine) or leukotriene receptor antagonist (montelukast) did not induce control of urticaria for the patient. Short oral corticosteroid (OCS) cures were repeatedly needed, followed by a rapid improvement of episodes of pruritus and induced urticaria. Considering the severe clinical course, we proposed therapy with anti-IgE monoclonal antibodies in order to obtain control of the disease and to reduce or eliminate the need for OCS therapy.

With the consent of the parents, we initiated the treatement with omalizumab $300 \mathrm{mg} / \mathrm{month}$, starting from an urticaria activity score for the preceding 7 days (UAS7) of 42 (Table I). Upon a promising improvement after the first three doses, the activity score of urticaria worsened again, thus at the end of the 5 doses of treatment, the UAS7 score was insignificantly modified (Fig. 1) and the patient and his family refused at this point to do the sixth dose of omalizumab from the schedule. Like in the previous case, the administration of omalizumab 
was very well tolerated, without side effects but the desired improvement of urticaria was not obtained for this patient. The lack of answer to recombinant monoclonal antibodies, the fear of adverse effects of other drugs (cyclosporine), determined the parents of the adolescent to refuse another option as add-on therapy and they decided to return to previous treatment schedule (non-sedating antihistamines, montelukast, OCS at need).

\section{Discussion}

The current guidelines on the treatment of urticaria state that the goal for the treatment of patients has to be complete symptom control. However, in many patients with physical urticaria, standard treatment with antihistamines is not sufficient to reach this goal. Following the recommended treatment algorithm, updosing of antihistamines should be performed in those patients with remaining symptoms. With this approach, many physical urticaria patients can be treated successfully. Nonetheless, there are some patients who still suffer from severe symptoms, despite updosing of $\mathrm{H} 1$ antihistamines and the addition of other medication such as leukotriene receptor antagonists and $\mathrm{H} 2$ receptor blockers. For these patients, alternative treatments such as cyclosporine A, dapsone or omalizumab therapy can be recommended. Omalizumab was approved by the US Food and Drug Administration and the European Medicines Agency for CSU. According to EACCI/ GA2LEN/EDF/WAO Guidelines, this drug is recommended as add-on therapy as a third line treatment for patient unresponsive to high doses of H1-antihistamines after 2-4 weeks or earlier $(2,3)$. Its action is to bind free serum $\operatorname{IgE}$ and prevent its attachement to the high-affinity receptor on mast cells and basophils. The effective doses in CSU are 150-300 mg/month and independent of total serum IgE level (4 quoted in 3). The good response in cold urticaria emphasizes the role of $\mathrm{IgE}$ in mast-cell activation by cold trigger. However, omalizumab has proven its effectiveness despite the level of total serum IgE, possibly because of other unknown effects (3).

The recommendation of anti-IgE treatment for patients with recalcitrant physical urticaria is supported by a recent small clinical trial and a number of case reports $(1,5,6)$. In our first patient, the observed very rapid improvement of the symptoms during omalizumab treatment argues in favour of the hypothesis that IgE plays an important role in the pathogenesis of physical urticarias. The kinetics of the relief from symptoms for this patient is similar to previously reported cases of inducible urticarias, as well as of chronic spontaneous urticaria, and strikingly different from the response in asthmatic patients which rarely show significant improvement within the first weeks.

This indicates that in asthma and urticaria, different pathophysiological mechanisms are responsible for the respective symptoms. In asthma, tissue remodelling in the lung and other 'chronic' processes are of pathophysiological importance which take much longer to be reversed. However, there might be currently unknown effects of omalizumab other than $\operatorname{IgE}$ capture, especially in regard to the inhibition of mast cell degranulation.

In terms of urticaria factitia, current data show that the majority of patients benefits from treatment with nonsedating antihistamines (7). The off-label use of treatment with omalizumab for the severe cases has already proven good results and there are data suggesting a better therapeutical answer to this drug in urticaria factitia compared with other forms of physical urticaria $(6,8)$. These encouraging data led us to start this treatment in our second patient with urticaria factitia. Unfortunately, the therapeutical response was unsatisfactory.

There are no parameters to select the responders from non-responders and in the literature the reported cases of omalizumab treated urticaria factitial, indicated that the patients with urticaria factitia and normal total $\operatorname{IgE}$ before starting the treatment are those with incomplete response of urticaria symptoms to omalizumab. In our case the baseline level of total IgE was increased (348 UI/ml), hence according to previously published results, we expected to have an improvement of symptoms for our patient. We could not explain the lack of response to the omalizumab treatment, so we can only speculate that perhaps different doses or different interval between the doses would have induced a better control of symptoms. Although there is no such recommendation in guidelines, there are authors reporting empirically use of regimens similar to asthma patients (doses calculated according to IgE level and weight) or doses between 150 and $300 \mathrm{mg}$ every 2 to 4 weeks, all this cumulated experience suggesting that the answer of CIndU to omalizumab might require a dose and administration regimen different from that recommended in CSU (9).

Unfortunately, there is not enough evidence in patient with CIndU, especially in AHs resistant cases and there are no criteria to identify the patients who would not profit from omalizumab treatment.

Clinical trials with larger patient numbers should be performed to further investigate the value of omalizumab, the individualized treatment dosages and administration intervals, and the biomarkers for future treatment algorithms in urticaria factitia and other forms of physical urticaria (9).

\section{Acknowledgements}

Not applicable.

\section{Funding}

No funding was received.

\section{Availability of data and materials}

The datasets used and/or analyzed during the current study are available from the corresponding author on reasonable request.

\section{Authors' contributions}

RSB contributed to the conception and design of the study. CGD was responsible for the conception of the study and contributed to drafting the manuscript. ECB contributed to the acquisition of the data. All authors read and approved the final manuscript.

\section{Ethics approval and consent to participate}

The study was approved by the Ethics Commitee of the Clinical Emergency Hospital for Children 'M.S. Curie' (Bucharest, 
Romania), and signed informed consent was obtained from the patients or the guardians included in this study.

\section{Patient consent for publication}

Signed informed consent was obtained from the patients or the guardians included in this study.

\section{Competing interests}

RSB and CGD declare that they have no conflicts of interest. ECB has participated in trials sponsored by Novartis.

\section{References}

1. Magerl M, Altrichter S, Borzova E, Giménez-Arnau A, Grattan CEH, Lawlor F, Mathelier-Fusade P, Meshkova RY, Zuberbier T, Metz M, et al: The definition, diagnostic testing, and management of chronic inducible urticarias - The EAACI/GA(2) LEN/EDF/UNEV consensus recommendations 2016 update and revision. Allergy 71: 780-802, 2016.

2. Zuberbier T, Aberer W, Asero R, Bindslev-Jensen C, Brzoza Z, Canonica GW, Church MK, Ensina LF, Giménez-Arnau A, Godse K, et al; European Academy of Allergy and Clinical Immunology; Global Allergy and Asthma European Network; European Dermatology Forum; World Allergy Organization: The EAACI/GA(2) LEN/EDF/WAO Guideline for the definition, classification, diagnosis, and management of urticaria: The 2013 revision and update. Allergy 69: 868-887, 2014.
3. Dressler C, Rosumeck S, Werner RN, Magerl M, Metz M, Maurer M, Nast A and Zuberbier T: Executive summary of the methods report for 'The EAACI/GA ${ }^{2} \mathrm{LEN} / \mathrm{EDF} / \mathrm{WAO}$ Guideline for the Definition, Classification, Diagnosis and Management of Urticaria. The 2017 Revision and Update'. Allergy 73: 1145-1146, 2018.

4. Saini S, Rosen KE, Hsieh HJ, Wong DA, Conner E, Kaplan A, Spector S and Maurer M: A randomized, placebo-controlled, dose-ranging study of single-dose omalizumab in patients with H1-antihistamine-refractory chronic idiopathic urticaria. J Allergy Clin Immunol 128: 567-573.e1, 2011.

5. Maurer M, Metz M, Brehler R, Hillen U, Jakob T, Mahler V, Pföhler C, Staubach P, Treudler R, Wedi B, et al: Omalizumab treatment in patients with chronic inducible urticaria: A systematic review of published evidence. J Allergy Clin Immunol 141: 638-649, 2018.

6. Chicharro P, Rodríguez P and de Argila D: Omalizumab in the treatment of chronic inducible urticaria. Actas Dermosifiliogr 108: 423-431, 2017 (In Spanish).

7. Krause K, Ardelean E, Kessler B, Magerl M, Metz M, Siebenhaar F, Weller K, Worm M, Zuberbier T and Maurer M: Antihistamine-resistant urticaria factitia successfully treated with anti-immunoglobulin E therapy. Allergy 65: 1494-1495, 2010.

8. Metz M, Ohanyan T, Church MK and Maurer M: Omalizumab is an effective and rapidly acting therapy in difficult-to-treat chronic urticaria: A retrospective clinical analysis. J Dermatol Sci 73: 57-62, 2014.

9. Uysal P, Eller E, Mortz CG and Bindslev-Jensen C: An algorithm for treating chronic urticaria with omalizumab: Dose interval should be individualized. J Allergy Clin Immunol 133: 914-915, 2014. 